ISSN 1659-3316

Especial: Profesores de Estudios Generales investigan

Dossier: El Quehacer de las Humanidades: Acción Social, Investigación y Metodologías e Innovación Tecnológica en la Docencia

\title{
Las Humanidades en la génesis de la regionalización universitaria: los Estudios Generales y el origen del proceso de regionalización de la Universidad de Costa Rica
}

\author{
Edgardo Fonseca Zúñiga \\ Recinto de Golfito, Universidad de Costa Rica, Costa Rica \\ edgardo.fonseca@ucr.ac.cr \\ https://orcid.org/0000-0001-6347-7533
}

Recibido: 14 de octubre de 2019

Aceptado: 15 de enero de 2020

Resumen: En 1968 inicia el proceso de regionalización de la Universidad de Costa Rica (UCR), con la creación de centros regionales universitarios en zonas alejadas del Valle Central. En esta investigación se analiza la labor cumplida por los Estudios Generales en este proceso. De esta manera se realizó una revisión de la expansión educativa de Costa Rica en las décadas de 1960 y 1970 y su relación con el modelo desarrollista impulsado por el Estado. Además se realizó una contextualización del modelo de educación superior aplicado en Centroamérica a partir la década de 1960, en donde la Educación General es fundamental. Finalmente se determina el papel jugado por los Estudios Generales como plataforma para impulsar la creación de centros que impartieran cursos universitarios más allá de la Ciudad Universitaria Rodrigo Facio. Las fuentes consultadas comprenden una extensa revisión bibliográfica e Informes de Rectoría.

Palabras clave: educación; enseñanza superior; Universidad; democratización de la educación; historia de la educación.

\section{(C) $(00$}

La Revista Estudios es editada por la Universidad de Costa Rica y se distribuye bajo una Licencia Creative Commons Atribución-NoComercial-CompartirIgual 3.0 Costa Rica. Para más información envíe un mensaje a 
Especial: Profesores de Estudios Generales investigan

\title{
The Humanities and the genesis of higher education regionalization: General Studies and the origin of the regionalization process of the University of Costa Rica
}

\begin{abstract}
In 1968, the process of regionalization of the University of Costa Rica (UCR) began, with the creation of regional university centers in remote areas of the Central Valley. This research analyzes the work accomplished by the General Studies in this process. In this way, a review of the educational expansion of Costa Rica in the 1960s and 1970s and its relationship with the state-driven developmental model was carried out. In addition, a contextualization of the higher education model applied in Central America was carried out from the 1960s, where General Education is fundamental. Finally, the role played by the General Studies as a platform to promote the creation of centers that offer university courses beyond the University City Rodrigo Facio is determined. The sources consulted include an extensive bibliographic review and Rectory Reports.
\end{abstract}

Keywords: education; higher education; Universities; democratization of education; educational history.

\section{Introducción}

En 1968 se inauguró el Centro Universitario Regional de San Ramón, con lo cual inicia el proceso de regionalización de la UCR. A partir de ese momento se fundaron otros centros universitarios en las provincias de Cartago, Guanacaste, Limón y Puntarenas. Aunque el proceso de creación de cada uno fue heterogéneo, tienen un factor en común: el impulso que significó para su apertura el impartir los cursos de Estudios Generales en comunidades fuera del Valle Central y brindar así a los estudiantes de estas regiones la oportunidad de acceder a la base humanista de las carreras de la UCR.

En esta investigación se analiza, desde un punto de vista histórico, la labor cumplida por los Estudios Generales y los cursos de Humanidades en el proceso de regionalización de la UCR. Para lograr el objetivo anterior se realizó una contextualización de la expansión educativa en Costa Rica en las décadas de 1960 y 1970 y su relación con el modelo desarrollista impulsado por el Estado.

\section{(c) (i) (2)}

La Revista Estudios es editada por la Universidad de Costa Rica y se distribuye bajo una Licencia Creative Commons Atribución-NoComercial-CompartirIgual 3.0 Costa Rica. Para más información envíe un mensaje a 
Especial: Profesores de Estudios Generales investigan

Seguidamente se determinó el papel jugado por la Educación General en el proceso de modernización de las universidades centroamericanas, contextualizado dentro del marco de la Guerra Fría. Finalmente se analizó el rol de los Estudios Generales como plataforma para impulsar la creación de centros que impartieran cursos universitarios más allá de la Ciudad Universitaria Rodrigo Facio.

Para cumplir con estos objetivos se realizó una amplia revisión bibliográfica sobre la expansión universitaria de la segunda mitad del siglo XX en Centroamérica, y sobre el proceso de creación de las sedes regionales de la UCR. Además, se consultaron fuentes primarias como Informes de Rectoría para analizar la posición de las autoridades universitarias en este proceso.

\section{Antecedentes de la expansión universitaria en Costa Rica en las décadas de 1960 y 1970}

\section{Desarrollismo y educación:}

El contexto histórico e institucional de Costa Rica en las décadas de 1960 y 1970 está ligado a la consolidación del proyecto desarrollista liderado, principalmente, por las administraciones de corte socialdemócrata del Partido Liberación Nacional (PLN). A nivel económico, este proyecto buscó implementar las medidas propuestas por la Comisión Económica para América Latina (CEPAL) referentes al modelo de sustitución de importaciones, el impulso a la industrialización del país y la tecnificación agrícola. En lo social, la política del PLN expandió y universalizó los servicios estatales, principalmente los referidos a salud, educación, asistencia social y acceso a agua potable y electricidad.

A nivel educativo, el sistema preuniversitario, que comprende preescolar, escuela y educación media (I, II y III ciclo, además de Educación Diversificada)

\section{(c) (i)(2)}

La Revista Estudios es editada por la Universidad de Costa Rica y se distribuye bajo una Licencia Creative Commons Atribución-NoComercial-CompartirIgual 3.0 Costa Rica. Para más información envíe un mensaje a 
Especial: Profesores de Estudios Generales investigan

sufre una transformación importante a raíz de la Ley General de Educación de 1973. Entre otros elementos, esta ley orienta los esfuerzos del país para universalizar el acceso a la escuela y aumenta la obligatoriedad de la educación al pasar del sexto grado (II ciclo) a los tres primeros años de enseñanza media (III ciclo) con lo cual es obligatoria la asistencia hasta 9 año de colegio'. Como resultado de esta política educativa, el país vivió una expansión sin precedentes en la apertura de centros educativos de segunda enseñanza. Esto tuvo dos consecuencias, la primera un aumento muy considerable en la cobertura de este nivel educativo durante esta década y la segunda, la conformación, cada vez mayor, de una población de graduados de bachillerato en enseñanza media, quienes presionaron por acceder a educación superior.

En este contexto es donde inicia la política de expansión de universidades públicas costarricenses. Desde 1940 el monopolio en esta materia se encontraba en la UCR, siendo durante tres décadas la única institución de educación superior, tanto pública como privada, que operó en el país. Inspiraba en el contexto de la Reforma del gobierno de Calderón Guardia (1940-1944), la UCR tuvo un rápido crecimiento desde su inauguración, proceso que benefició principalmente a hombres, capas urbanas y clase media- altaii. Así, para finales de la década de 1960 se expresa la restringida capacidad que tiene para absorber a la creciente población graduada de secundaria. Para esta época el $90 \%$ de su población estudiantil es originaria del Valle Central, lo que evidencia que el acceso de estudiantes de zonas costeras y otras regiones del país era muy limitado iii.

Dentro del contexto estudiantil mencionado previamente, cabe mencionar un fenómeno cultural de importancia para finales de 1960 e inicios de 1970. Con la instauración en la UCR del ciclo de Estudios Generales, obligatorios para toda carrera desde la Reforma Universitaria de 1957, la UCR afianzó su modelo

\section{(ब) (O०)}

La Revista Estudios es editada por la Universidad de Costa Rica y se distribuye bajo una Licencia Creative Commons Atribución-NoComercial-CompartirIgual 3.0 Costa Rica. Para más información envíe un mensaje a 


\section{Especial: Profesores de Estudios Generales investigan}

humanista ${ }^{1}$. La maduración de estos Estudios Generales va de la mano con la radicalización de la juventud universitaria, pues la discusión política y filosófica de estos cursos significó la primera ventana de muchos de ellos a una visión crítica de la actualidad nacional y mundial. Igualmente, el cambio curricular de 1966 implicó la impartición de filosofía marxista, el estudio del proceso histórico del trabajo, la creación de la clase obrera y la realidad latinoamericana, enfocada en el imperialismo estadounidense ${ }^{\text {iv }}$.

Valiosos testimonios a propósito de este fenómeno fueron recopilados por la Revista Estudios de la Escuela de Estudios Generales en 2015 a propósito del 75 aniversario de la fundación de la UCR. Por ejemplo, el historiador Vladimir de la Cruz de Lemos recuerda que:

"Había sucedido un acontecimiento especial, que era que para 1967, por una lucha muy importante que habían dado profesores como Teodoro Olarte, Constantino Láscariz, Víctor Manuel Arroyo, y otros, a partir de este año, 1967, se empezaría con un nuevo programa de estudios que se concentraría en el estudio del Siglo XIX, que implicaba la Revolución Industrial, el Romanticismo, las corrientes políticas nuevas, el socialismo, el comunismo, el anarquismo, la proyección de la Revolución Francesa en las grandes revoluciones europeas de 1820, 1830 y 1848, la independencia americana y la lucha anticolonial americana, el estudio de personajes como Compte (sic), Hegel, Marx, Engels, y otros filósofos, el estudio del Manifiesto Comunista, como uno de los documentos más importantes de ese siglo, entre otras cosas. Esto nos permitió a los estudiantes de 1967 impulsar un gran debate político sobre la contemporaneidad."

De igual manera la abogada Zarela Villanueva Monge recuerda su experiencia en Estudios Generales de la siguiente manera:

"Fue una década donde las amenazas de la guerra fría resultaban un tema cotidiano; los países latinoamericanos se debatían en cruentos procesos dictatoriales; y los desafíos contra la democracia también tambaleaban en nuestras fronteras. En las aulas de Estudios Generales se cimentaron las primeras discusiones serias y adultas de quienes empezábamos a ser protagonistas de un

\footnotetext{
${ }^{1}$ Este proceso de creación y consolidación de los Estudios Generales en la UCR se detalla en el siguiente apartado.
}

La Revista Estudios es editada por la Universidad de Costa Rica y se distribuye bajo una Licencia Creative Commons Atribución-NoComercial-CompartirIgual 3.0 Costa Rica. Para más información envíe un mensaje a revistaestudios.eeg@ucr.ac.cr. 


\section{Especial: Profesores de Estudios Generales investigan}

fuerte movimiento estudiantil caracterizado por los ideales de paz y de prosperidad para todos y todas, que abanderamos en esa época."vi

Un análisis de estos dos testimonios evidencia la influencia política de los cursos de Estudios Generales en esta generación radicalizada. De la Cruz afirma que la representación estudiantil que ayudó a conformar, el Frente de Acción Universitaria (FAU) tenía su principal zona de influencia en estudiantes de Estudios Generales, además de organizar distribuciones gratuitas del Manifiesto Comunista y la edición de un periódico estudiantil titulado Unidadvii. Villanueva confirma que las discusiones en las aulas y su visión humanista fue lo que contribuyó a toda una generación a formar una visión más crítica de la actualidad de ese momentoviii.

La ponencia "Estudiar y Luchar" en el II Congreso Universitario en 1966 a propósito del papel de la participación estudiantil, la lucha contra el contrato-ley favorable a la empresa minera ALCOA en 1970, la reformulación de la Federación de Estudiantes de la UCR (FEUCR) y la exigencia de mayor participación estudiantil en el gobierno universitario, son expresiones concretas de este proceso $^{\mathrm{ix}}$.

La base de esta radicalización, la implementación de Estudios Generales al inicio de la carrera universitaria, fue una política educativa aplicada en toda Centroamérica ${ }^{2}$. Al igual que ocurrió en Costa Rica, estudiantes de Guatemala y El Salvador sufrieron procesos de politización significativos ${ }^{3}$. Para Guatemala estudiantes de Estudios Básicos de la Universidad de San Carlos (USAC) protagonizaron una importante huelga en $1969^{x}$ y en El Salvador, estudiantes de Áreas Comunes de la Universidad de El Salvador (UES) protagonizaron una huelga en $1970^{x i}$. En ambos casos la principal motivación fue el restringido acceso

\footnotetext{
${ }^{2}$ Detalles de este proceso a nivel centroamericano se brindan en el siguiente apartado.

${ }^{3}$ Agradezco al profesor Arturo Tarracena Arriola y al Ing. Ricardo Trujillo Molina quienes en distintas conversaciones me compartieron valiosos datos acerca de sus experiencias durante este periodo en la Universidad de San Carlos de Guatemala y en la Universidad de El Salvador respectivamente.
}

\section{(c) (1) 8 ()}

La Revista Estudios es editada por la Universidad de Costa Rica y se distribuye bajo una Licencia Creative Commons Atribución-NoComercial-CompartirIgual 3.0 Costa Rica. Para más información envíe un mensaje a revistaestudios.eeg@ucr.ac.cr. 


\section{Especial: Profesores de Estudios Generales investigan}

a carreras de gran demanda como Medicina y Derecho y el filtro que significaban estos cursos básicos para el avance en los estudios universitarios, pues muchos estudiantes provenientes de secundarias públicas encontraban un importante desface entre la ciencia que aprendieron en esas aulas y la que les enseñaban en la universidad ${ }^{\mathrm{xii}}$. Para el caso salvadoreño esta radicalización también significó una militancia política, pues del Comité de Representantes de Áreas Comunes (CRAC) se derivaron los Comandos Organizadores del Pueblo (COP) un grupo de estudiantes universitarios vinculados posteriormente al Ejército Revolucionario del Pueblo (ERP) xiii.

Este proceso cultural y político debe tomarse en cuenta para comprender el proyecto de creación de nuevos centros universitarios llevado a cabo en Costa Rica a partir de la década de 1970. A la par de promover la urgente modernización de la UCR, la política educativa de la década se orientó a la creación de nuevas universidades estatales que cubrieran la demanda en educación superior. Además, estas universidades debían tener una oferta académica orientada a cubrir las necesidades que el sistema económico y la expansión social del Estado requerían. En síntesis, las necesidades económicas de la industrialización, la tecnificación, la especialización agrícola y la expansión de servicios como educación y salud crearon circunstancias en las cuales una rebasada UCR no estaba en condiciones de afrontar. La solución surgió del mismo Estado costarricense al reconfigurar la educación superior estatal del país.

Para analizar este proceso, primero observaremos el proceso de creación de nuevas universidades y posteriormente la reconfiguración de la UCR, en donde el programa de regionalización se convierte en un eje central de su modernización. Con un ambiente político favorable, el PLN gobernaba con una mayoría en la Asamblea Legislativa, este partido pudo materializar sus proyectos educativos con relativa facilidad. La primera universidad nueva en entrar en funciones fue la Universidad Nacional (UNA) en marzo de 1973, situada en la ciudad de Heredia.

\section{(c) (i) (2)}

La Revista Estudios es editada por la Universidad de Costa Rica y se distribuye bajo una Licencia Creative Commons Atribución-NoComercial-CompartirIgual 3.0 Costa Rica. Para más información envíe un mensaje a 
Especial: Profesores de Estudios Generales investigan

Esta se creó sobre la Escuela Normal, la institución encargada de la formación de maestros en el país desde su refundación en 1950. Con este antecedente, el objetivo de la nueva universidad fue claro: una institución con orientación pedagógica para formar los docentes necesarios para enfrentar la expansión educativa que estaba teniendo lugar ${ }^{\text {xiv }}$. La UNA nació regionalizada, al tomar como parte de sus funciones las sedes de la Normal en Liberia, Pérez Zeledón y San Ramón.

La segunda de las universidades fundada fue el Instituto Tecnológico de Costa Rica (ITCR) en ese mismo año. Esta institución fue creada en la provincia de Cartago, con una vigorosa participación comunal en su proceso de creación. El ITCR se orientó a carreras técnicas y de duración intermedia. El objetivo es nuevamente claro, impulsar la creación y especialización de la mano de obra que el país necesitaba para suplir el modelo económico de sustitución de importaciones y la incipiente industrialización ${ }^{\mathrm{xv}}$. Como ocurre con la UNA, el ITCR también nace regionalizado, al absorber la Escuela Técnica Nacional en San José y la Escuela Nacional de Agricultura en San Carlos.

El tercero de estos centros educativos superiores creados fue la Universidad Estatal a Distancia (UNED) en 1977. Este implementó el modelo educativo a distancia, teniendo como público meta a habitantes de la zona rural, clase obrera, amas de casas, población penitenciaria e indígenas ${ }^{x v i}$. Las cuatro universidades estatales coordinan su política educativa y su relación con el Estado a través del Consejo Nacional de Rectores (CONARE) creado en 1974.

El común denominador, a nivel político, de estas nuevas universidades es la enorme intervención del Poder Ejecutivo en sus gobiernos. Así estos centros fueron creados sin una autonomía análoga a la aplicada en la UCR, con lo cual su dirección estaba fuertemente influida por la línea política oficialista. Los ejemplos son concretos: la primera rectoría de la UNA y su formación ideológica recayeron en el sacerdote Benjamín Núnez, una prominente figura liberacionista. En el caso

\section{(c) (i)(2) (2)}

La Revista Estudios es editada por la Universidad de Costa Rica y se distribuye bajo una Licencia Creative Commons Atribución-NoComercial-CompartirIgual 3.0 Costa Rica. Para más información envíe un mensaje a revistaestudios.eeg@ucr.ac.cr. 
Especial: Profesores de Estudios Generales investigan

del ITCR su gobierno recaía en una junta administrativa con representación del Ministerio de Agricultura y el Ministerio de Economía, Industria y Comercio. La UNED contó entre sus fundadores a figuras centrales del PLN como Francisco Antonio Pacheco.

Con esta intervención del Poder Ejecutivo tan acentuada, las nuevas universidades serían centros destinados a "recuperar" un paraíso perdido en la UCR, donde la radicalización política antes descrita había formado movilizaciones estudiantiles considerables ${ }^{\text {xvii. }}$ Igualmente, esta intervención directa en el gobierno de las nuevas universidades garantizaba que sus acciones se plegaran por completo al proyecto político socialdemócrata. La restauración de ese paraíso perdido fue momentánea, pues movimientos huelguísticos estudiantiles pronto cuestionaron el poder del rector Núñez en la UNA y en el ITCR fuertes huelgas logran democratizar esta institución entre 1980 y $1982^{\text {xviii }}$.

\section{Desarrollismo y regionalización:}

El caso de la UCR merece una especial atención. Esta universidad celebró su III Congreso Universitario en 1971, siendo este el foro en donde se discutieron las principales necesidades y los cambios más urgentes. La modernización necesaria para esta institución pasó por un objetivo claro, lograr un mayor vínculo universidad - sociedad. De esta manera se buscó superar el modelo profesionalista, que limitaba las labores de la universidad a generar títulos, y se intentó un modelo universitario orientado a actividades sustantivas que involucraran a la Universidad en la dinamización de la sociedad. Esto se logró con la fundación de la Vicerrectoría de Investigación, la Vicerrectoría de Acción Social y el Trabajo Comunal Universitarioxix.

En el gobierno universitario se logran importantes reformas asociadas a una democratización de las autoridades. De esta forma, se reconfigura el Consejo Universitario con funcionarios elegidos periódicamente representando las áreas

\section{(C) $(\triangle \odot)$}

La Revista Estudios es editada por la Universidad de Costa Rica y se distribuye bajo una Licencia Creative Commons Atribución-NoComercial-CompartirIgual 3.0 Costa Rica. Para más información envíe un mensaje a 


\section{Especial: Profesores de Estudios Generales investigan}

académicas y administrativas de la Universidad. Igualmente se logra la participación estudiantil en los órganos colegiados como son el mismo Consejo Universitario y las Asambleas de cada facultad y escuela ${ }^{\mathrm{xx}}$.

En materia de cobertura, las reformas de la UCR involucraron una expansión de matrícula sustanciosa, logrando un crecimiento significativo en un tiempo corto. Es en este punto donde toma gran importancia el programa de regionalización, con el cual la UCR funda sedes a lo largo del territorio nacional. Impulsado desde 1968 por el rector Carlos Monge Alfaro, la regionalización universitaria inició como un proyecto para impartir carreras cortas y profesionalizar a los docentes en servicio. En una década, de 1968 a 1979, se fundaron cinco sedes en cinco provincias, incluyendo tres de ellas en regiones costeras. EI III Congreso introduce en el Estatuto Orgánico el concepto y la reglamentación de estos centros regionales ${ }^{\mathrm{xxi}}$.

Contextualizado este elemento, es importante establecer la relación existente entre la regionalización universitaria y el modelo desarrollista que impulsa toda la reestructuración universitaria en el país. Como ha sido citado, la transformación del modelo productivo y la expansión de servicios estatales marcan este periodo y la regionalización universitaria es un instrumento para dinamizar este proceso.

El Informe del Rector brindado por Carlos Monge Alfaro en el año 1968, dedica el segundo capítulo a plantear la relación entre la Universidad y desarrollo regional. En esta sección, Monge Alfaro habla sobre la expansión educativa surgida en la Costa Rica rural, en la cual "son muchos los liceos creados en las diferentes comunidades, muchas las inquietudes surgidas en los hogares humildes, de escasos recursos económicos y las ambiciones de cientos de adolescentes que desean continuar estudios superiores"xii. Monge Alfaro interpreta que esta política educativa traspasa nuevas responsabilidades a la

\section{(c) (i) (2)}

La Revista Estudios es editada por la Universidad de Costa Rica y se distribuye bajo una Licencia Creative Commons Atribución-NoComercial-CompartirIgual 3.0 Costa Rica. Para más información envíe un mensaje a 
Especial: Profesores de Estudios Generales investigan

Universidad, la cual debe tomar la responsabilidad de garantizar que esos nuevos bachilleres obtengan un título.

Para lograr este objetivo, el rector sugiere colocar en sintonía la política de desarrollo comunal del gobierno de Trejos Fernández, con el proyecto de regionalización universitaria. A propósito menciona:

"La política del Gobierno del Presidente (sic) Trejos Fernández tendientes a estimular a las comunidades, a seleccionar a sus mejores valores espirituales y sociales para que adquieran conciencia de su propio poder y colaboren en el proceso de desarrollo nacional -educativo, económico y social- parece signo de un nuevo rumbo histórico, de una actitud positiva y consciente para elevar el nivel de cultura y la aptitud creadora de las regiones lejanas. La Universidad de Costa Rica como respuesta a esa política y además porque desde hace años en conferencias y en congresos universitarios se clama por acercarse al pueblo para conocerlo y servirle mejor, ha pensado en poner su grano de arena con la creación de Centros Universitarios Regionales." "xiii

Esta tendencia a sintonizar ambas políticas también queda evidenciada en el discurso que brinda en Limón en 1969, a propósito de la celebración de la sesión del Consejo Universitario en esta ciudad. El rector comenta que, ante la petición de las autoridades limonenses de la instalación de un centro regional universitario en la ciudad, este debe planificarse de acuerdo con las necesidades y políticas de desarrollo de la región ${ }^{x x i v .}$

La posición de las autoridades universitarias era clara sobre el papel de la regionalización universitaria con respecto al desarrollismo impulsado desde el gobierno. Observemos ahora dos casos concretos que ilustran lo anterior. La expansión educativa generada en el país desde la segunda mitad del siglo XX significó que el sistema se enfrentara a una severa crisis en la formación de docentes. La reactivación de la Escuela Normal en 1950, con sus respectivas sedes regionales, además de la creación de la Escuela Normal Superior en 1966, fueron intentos desde el Ejecutivo para paliar dicha situación ${ }^{\mathrm{xxv}}$. Dentro de los esfuerzos de la UCR para suplir de maestros al país resulta particularmente

\section{(c) (i) (2)}

La Revista Estudios es editada por la Universidad de Costa Rica y se distribuye bajo una Licencia Creative Commons Atribución-NoComercial-CompartirIgual 3.0 Costa Rica. Para más información envíe un mensaje a 
Especial: Profesores de Estudios Generales investigan

interesante el primer proyecto de regionalización universitaria presentado por la entonces decana de la Facultad de Educación, María Eugenia Dengo, en el año de 1967.

El objetivo de este proyecto salta a la vista en su título: "Algunas ideas sobre el establecimiento de Escuelas o Colegios Universitarios Regionales con aprovechamiento de las Escuelas Normales existentes"xxvi. Estos centros tendrían como principal misión la especialización de docentes que estuvieran en servicio y que por razones de distancia no pudieran asistir a la sede central de la Universidad, además de ofrecer una carrera corta de educación para preparar en un breve plazo a docentes y capacitarlos para que ejercieran el magisterio en el menor tiempo posible. Para llevar a cabo este plan, Dengo proponía un enriquecimiento de los planes de estudio ya existentes en la Escuela Normal, coordinados con el Ministerio de Educación Pública (MEP), en donde se incluyeran cursos de Estudios Generales y cursos de especialización. Los detalles de esta propuesta se observan en la próxima sección, por el momento es suficiente señalar que este primer proyecto de regionalización universitaria intentó ser una respuesta a un problema derivado del nuevo modelo político y económico del país.

Otro ejemplo claro es el referido a la creación de un centro regional universitario en la provincia de Guanacaste. Desde 1968, la creación del Centro Regional de Liberia está impulsada por la idea de dotar a esta provincia de un lugar para la formación de peritos agropecuarios ${ }^{\mathrm{x} v \mathrm{ii}}$. Este plan buscaba poner en sintonía la tecnificación agrícola vivida en el país con la regionalización universitaria. De esta manera, el proyecto original era la implementación de carreras cortas en la rama agropecuaria. El motivo del atraso de la apertura de este centro regional se encuentra en un desacuerdo entre el Gobierno Central, por medio del Ministerio de Agricultura y Ganadería (MAG), con la UCR. Este conflicto gira en torno al lugar en donde debía ser colocada una escuela agrícola 
Especial: Profesores de Estudios Generales investigan

recomendada por la Agencia Internacional de Desarrollo de Estados Unidos (USAID). Este ente proponía un modelo simlar al aplicado en la Escuela Zamora en Honduras, con una fuerte intervención del Poder Ejecutivo, situación que la UCR, apelando a su autonomía, quería evitar. La exclusión de la UCR en este plan de escuelas agrícolas y el temor a duplicar esfuerzos, atrasaron la apertura del centro regional liberiano.

Estos dos ejemplos vinculan la creación de centros regionales con el modelo político y económico de desarrollo implementado en esta época. Lo anterior queda ilustrado en las palabras de la decana Dengo:

"Las carreras cortas de tipo técnico, así como las carreras de magisterio de enseñanza primaria y media serían quizás los prototipos de profesiones que mediante la instalación de colegios regionales universitarios podrían ofrecerse para satisfacer las demandas crecientes en estos campos."xxviii

El rector Monge Alfaro interpretó esta nueva tarea de la Universidad desde el desarrollismo. Junto al discurso sobre democracia e igualdad de oportunidades, da a la regionalización un papel en la distribución de la riqueza y en el desarrollo integral de la sociedad, dentro de una visión que observa en las universidades motores de desarrollo, puntos de integración nacional y suplidores de las demandas tanto económicas como culturales de la sociedad.

El posterior desarrollo del proyecto de regionalización de la Universidad de Costa Rica desbordó este planteamiento original. Los Centros Regionales abarcaron tareas más allá de las primeramente planteadas por sus fundadores y el papel jugado por los Estudios Generales en este proceso es analizado a continuación.

\section{(C) $(00$}

La Revista Estudios es editada por la Universidad de Costa Rica y se distribuye bajo una Licencia Creative Commons Atribución-NoComercial-CompartirIgual 3.0 Costa Rica. Para más información envíe un mensaje a 


\section{Regionalizando los Estudios Generales}

\section{Especial: Profesores de Estudios Generales investigan}

\section{La Educación General en el contexto centroamericano y la Guerra Fría:}

El proyecto de una Educación General planificada como un conjunto de estudios básicos al inicio de la carrera universitaria, con un enfoque humanistas y orientados al aprendizaje de elementos de las ciencias sociales y básicas, tiene su origen en el sistema educativo estadounidensexxix. Este modelo fue impulsado desde la década de 1920 y después de la Segunda Guerra Mundial, en el ambiente de Guerra Fría, fue exportado a la Cuenca del Caribe, teniendo en la Universidad de Puerto Rico a su primer aliado y difusor. De esta manera, esta Educación General se proyectó como parte integral de todo un proyecto para modernizar las universidades centroamericanas.

En Costa Rica este proceso avanzó en 1957 con la Reforma Universitaria, la inauguración de la Facultad de Ciencias y Letras y la puesta en marcha el programa de Estudios Generales. Después de dos proyectos fallidos para intentar reformar a la UCR, presentados entre 1946 y 1948, el tercer plan impulsado desde 1954 por Rodrigo Facio Brenes y Carlos Monge Alfaro logró consolidarse, estableciendo como objetivo principal brindar una educación integradora, más allá de la simple especialización, en donde el estudiante universitario tuviera contacto con conocimientos científicos distintos de su área de estudio ${ }^{\mathrm{xx}}$. El símbolo de esta reforma fue la mencionada Facultad de Ciencias y Letras, lugar en donde fue subordinado el Departamento de Estudios Generales, estableciendo que:

"Todos los alumnos de la Universidad debían cursar en el Departamento de Estudios Generales tres materias comunes y una optativa. Las materias comunes serían: Filosofía, Historia y Castellano; las optativas debían elegirse entre Biología, Sociología y Matemáticas, en el entendido de que los alumnos en cuyo plan de estudios tuvieren una de esas materias optativas debían de elegir otra entre las restantes."

\section{(c) (i) (2)}

La Revista Estudios es editada por la Universidad de Costa Rica y se distribuye bajo una Licencia Creative Commons Atribución-NoComercial-CompartirIgual 3.0 Costa Rica. Para más información envíe un mensaje a 


\section{Especial: Profesores de Estudios Generales investigan}

Sumado a lo anterior, el estudiante llevaba un curso de elaboración de una investigación científica y otras materias de ciencias básicas, ciencias sociales, artísticas y una actividad deportiva, todo con el objetivo de conseguir una educación integral. Este proceso era denominado Ciclo de Educación Básica y debía ser aprobado por todo estudiante de la UCR ${ }^{\text {xxxii }}$.

La ruta costarricense continúa con la firma del Convenio de Intercambio entre la Universidad de Costa Rica y la Kansas University (KU) en 1958, el cual permitió, entre otros aspectos, la operación en el país de la Carnegie Foundation y los Cuerpos de Paz a inicios de la década de 1960 xxxiii. Estos organismos son fundamentales para poner en marcha el plan cultural estadounidense en el sistema educativo nacional. Particularmente los Cuerpos de Paz realizaron sesiones de capacitación a los docentes de escuelas y colegios, por medio de la Facultad de Educación.

Este proceso estuvo contextualizado dentro del impulso para sistematizar un modelo universitario autónomo y humanista, con su base educativa en la Educación General, en el resto de las universidades centroamericanas. Así, en los esfuerzos por lograr autonomía debe destacarse la declaratoria de esta en la USAC en Guatemala en $1944^{\text {xxxiv }}$, en la Universidad Autónoma de Honduras (UNAH) en $1957^{x \times x v}$ y la elevación a rango constitucional de la autonomía de la Universidad Nacional de León en Nicaragua en $1966^{\text {xxxvi }}$.

En el campo del humanismo y la Educación General, la USAC inició con la implementación de los Estudios Básicos en 1961 y la creación de la Escuela de Estudios Generales en 1966. Esta operó hasta 1968, pues tanto la oposición estudiantil como la competencia de la Facultad de Humanidades suprimieron sus actividades $^{\mathrm{xx} x \mathrm{vii}}$. En El Salvador se dio la implementación del programa de Áreas Comunes en la UES durante la Reforma Universitaria de 1963. Así la Facultad de Humanidades, creada desde 1948, es transformada en una Facultad de Ciencias y Humanidades, asignándole la tarea de impartir los cursos de humanidades,

\section{(c) (i) (9) (2)}

La Revista Estudios es editada por la Universidad de Costa Rica y se distribuye bajo una Licencia Creative Commons Atribución-NoComercial-CompartirIgual 3.0 Costa Rica. Para más información envíe un mensaje a 
Especial: Profesores de Estudios Generales investigan ciencias sociales y ciencias básicas, conformando las Áreas Comunes. Este proceso se acompañó de una democratización de la UES con un aumento de matrícula, la construcción de comedores y residencias estudiantiles, además de la Biblioteca Central y los centros regionales de Santa Ana y San Miguel ${ }^{x x v i i i}$. Por la radicalización estudiantil antes citada y el endurecimiento de la política anticomunista del gobierno, este proceso es finalizado de manera violenta con la intervención militar de 1972 y el exilio, en muchos casos hacia Costa Rica, de las principales autoridades universitarias.

En Honduras, después de la declaratoria de la autonomía, la UNAH inicia un proceso de reforma fuertemente respaldado por los estudiantes agrupados en el Frente de Reforma Universitaria (FRU). En 1960 inicia el proyecto de Reforma Universitaria con la creación de un Departamento de Ciencias Básicas, el cual fue transformado posteriormente en el Centro Universitario de Estudios Generales (CUEG), con la misión de impartir cursos de humanidades y ciencias a todos los estudiantes. Además en ese mismo año funcionó la Comisión de Reforma Universitaria, la cual fue disuelta por el golpe de estado de $1963^{\text {xxxix }}$.

Desde un punto de vista filosófico e ideológico este proceso fue impulsado por la UNESCO y su plan para modernizar la educación superior latinoamericana. Desde un punto de vista administrativo, estuvo coordinado por el Consejo Superior de Universidades Centroamericanas (CSUCA), siendo la primera política de integración educativa llevada a cabo por esta institución. Creado en 1948 en San Salvador, este ente tuvo un importante impulso con las políticas integradoras centroamericanas de la década de 1950, como son la Organización de Estados Centroamericanos (ODECA) y el Mercado Común Centroamericano (MCCA). Para 1959 se crea la Secretaría Permanente en San José, Costa Rica y se edita el Primer Plan para la Integración Regional de la Educación Superior Centroamericana (PIRESC I), el cual busca armonizar el modelo educativo de la región por medio de la Educación General, además de promover la

\section{(c) (i) (9) (2)}

La Revista Estudios es editada por la Universidad de Costa Rica y se distribuye bajo una Licencia Creative Commons Atribución-NoComercial-CompartirIgual 3.0 Costa Rica. Para más información envíe un mensaje a 
Especial: Profesores de Estudios Generales investigan departamentalización de estos centros de estudio y la construcción de ciudades universitarias ${ }^{\mathrm{xl}}$.

El CSUCA se convirtió en la institución por la cual Estados Unidos extendería su influencia cultural en el área centroamericana. Por medio de la Oficina Regional para Centroamérica y Panamá (ROCAP) y aprovechando el acercamiento entre la UCR y la KU, las agencias de cooperación y financiamiento estadounidenses ejercieron una importante influencia sobre el nuevo proyecto de educación superior de la región. Capacitaciones, reuniones y convenios hicieron que la Fundación Ford, Carnegie Foundation, el Banco Interamericano de Desarrollo (BID), la USAID y la Alianza para el Progreso participaran activamente en el financiamiento y desarrollo de las reformas universitarias centroamericanas ${ }^{x i}$. A modo de ejemplo, la Ciudad Universitaria en San Salvador contó con un importante apoyo financiero del BID y la Fundación Rockefeller rlii.

La contradicción entre la influencia estadounidense en este proceso y la radicalización, en muchos casos antiimperialista, de los estudiantes que la disfrutaron, plantea una paradoja que ya advertía Isaac Felipe Azofeifa, director de la Escuela de Estudios Generales, cuando evaluaba los acontecimientos de este periodo en 1974:

"Algunos argumentan que los Estudios Generales fueron introducidos como clausula obligatoria por los prestamistas norteamericanos del BID y la Alianza para el Progreso, para afirmar nuestra colonización y dependencia cultural. Si fue así "les salió la venada careta". Erraron el tiro, por lo menos en la experiencia de la Universidad de Costa Rica."

Los Estudios Generales en la UCR fueron modificados de manera significativa después del III Congreso Universitario. En 1974 la Facultad de Ciencias y Letras fue abolida y se creó la Escuela de Estudios Generales, la cual tomó la dirección de los Cursos Integrados de Humanidades, los recién creados Seminarios de Realidad Nacional y los cursos optativos en modo de repertorio.

\section{(c) (i) (2)}

La Revista Estudios es editada por la Universidad de Costa Rica y se distribuye bajo una Licencia Creative Commons Atribución-NoComercial-CompartirIgual 3.0 Costa Rica. Para más información envíe un mensaje a revistaestudios.eeg@ucr.ac.cr. 
Especial: Profesores de Estudios Generales investigan

Además se introdujo, por influencia del profesor chileno Raúl Torre, los Seminarios Participativos, una opción inspirada en el modelo de cogestión pedagógica de

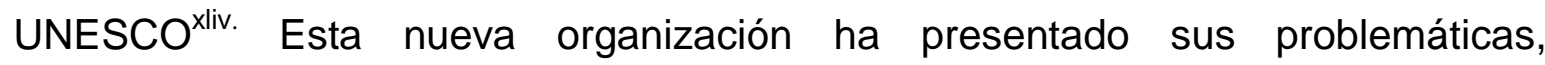
relacionadas con un debilitamiento de la institucionalidad de los Estudios Generales y una disminución en su peso en los planes de estudio de las distintas carreras universitarias ${ }^{\mathrm{xlv}}$.

\section{Estudios Generales y regionalización:}

Para comprender lo anterior se debe contextualizar la dinámica de admisión y la entrada a una carrera en la UCR de las décadas de 1960 y 1970. A partir de 1960 la Universidad aplicó un examen de admisión que combinaba la nota obtenida por el estudiante junto con su desempeño en el examen de

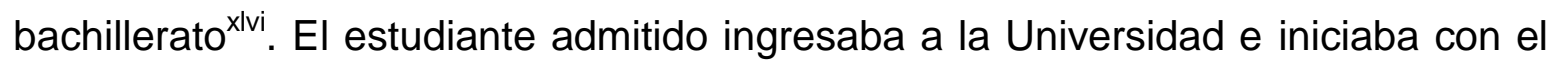
Ciclo de Educación Básica antes descrito, lo que comúnmente se denominaba precarrera. Una vez que lo aprobaba, proceso que debía culminar en cuatro semestres, en su tercer año universitario ingresaba a carrera. Cuando la demanda de carreras aumentó, estas se dividieron en dos secciones: 1- las carreras sin cupo, las cuales el estudiante podía entrar libremente y 2- las carreras con cupo, en donde existía una restricción de ingreso y el estudiante debía contar con requisitos (promedio ponderado, examen de actitud) para ingresar. Este proceso tenía al menos dos objetivos, por un lado garantizar una formación integral en el estudiante antes de su especialización y en segundo lugar ubicar vocacionalmente al alumno, antes de tomar la decisión de que carrera cursar.

Este proceso se mantuvo hasta finales de la década de 1970, cuando las autoridades universitarias introdujeron cambios significativos en el sistema de matrícula. Este cambio se impulsó principalmente por dos motivos, el primero fue el compromiso adquirido en el III Congreso Universitario de estabilizar la matrícula en aproximadamente 24 mil estudiantes y el segundo era disminuir la cantidad de

\section{(c) (i)(2)}

La Revista Estudios es editada por la Universidad de Costa Rica y se distribuye bajo una Licencia Creative Commons Atribución-NoComercial-CompartirIgual 3.0 Costa Rica. Para más información envíe un mensaje a 


\section{Especial: Profesores de Estudios Generales investigan}

alumnos que cursaban durante varios años materias de Educación Básica y no lograban colocarse en ninguna carrera, cifra que llegó a ser de 4191 alumnos hacia $1977^{\text {xlvii }}$. Por lo tanto, las autoridades se impusieron como meta lograr una nivelación de admitidos y estudiantes matriculados y así regular la cantidad total de alumnos. De esta manera, en un proceso no exento de polémica, en 1975 se abolió la precarrera para los estudiantes nuevos y todo ingresado debía matricular directamente en una carrera universitaria ${ }^{\text {xliii }}$, configurando los Estudios Generales al plan de estudios de cada una.

En este punto es pertinente preguntar, todo este proceso ¿cómo se manifiesta en los Centros Regionales? ¿de que manera las autoridades universitarias compaginaron la formación en Educación Básica con las carreras cortas propuestas en estos centros? Este elemento que se considera identitario de la UCR ¿cómo se adaptó al nuevo proyecto educativo concretizado en la regionalización? Como ha sido expuesto, los Centros Regionales tienen su génesis en un plan educativo con objetivos muy concretos. Los centros aprobados por el Consejo Universitario en 1968 en San Ramón y Liberia desarrollaban un programa muy preciso al implementar carreras cortas en educación y agronomía respectivamente. Ante este paradigma ¿cómo se adaptaron los Estudios Generales a esta nueva modalidad?

Las primeras respuestas a estas interrogantes se pueden observar en la discusión de las funciones de los centros regionales realizadas en el Consejo Universitario. La propuesta discutida en 1967 fue la siguiente. Se crearía un nuevo programa en donde el estudiante cursaría un Ciclo Básico de Ciencias y Letras, similar al ofrecido en la Facultad de Ciencias y Letras de la sede central. Este ciclo tendría cursos en las áreas de Humanidades, Ciencias Sociales, Ciencias Físico Matemáticas y Biología. Aprobada esta formación básica, el estudiante obtenía un Certificado de Educación Básica y podía continuar la carrera corta en el centro regional o trasladarse a la Ciudad Universitaria Rodrigo Facio a optar por una de

\section{(C) $(00$}

La Revista Estudios es editada por la Universidad de Costa Rica y se distribuye bajo una Licencia Creative Commons Atribución-NoComercial-CompartirIgual 3.0 Costa Rica. Para más información envíe un mensaje a 


\section{Especial: Profesores de Estudios Generales investigan}

sus carreras. Este ciclo básico era la base del llamado Programa de Transferencia, al cual optarían los estudiantes que desearan aplicar dicho traslado ${ }^{\mathrm{xlix}}$.

La discusión del Consejo Universitario es interesante en este punto. Aún cuando la defensa de Monge Alfaro y la comisión sobre regionalización es la creación de centros que gradúen a estudiantes en sus propias carreras, la observación hecha por Oscar Ramírez acerca de convertir estos centros regionales en espacios para obtener el ciclo de Educación Básica y así desahogar la matrícula de la sede central, saltando "el cuello de botella" del primer año universitario, es relevantel. Aunque fue objetada por los otros miembros del Consejo, el avance de la década de 1970 parece confirmar que, al menos en parte, los centros regionales realmente llegaron a cumplir esa función.

Otra propuesta nació desde la Rectoría, apoyada por Carlos Monge Alfaro en 1968. De esta manera, este funcionario recomendaba que los nuevos centros regionales tuvieran planes y programas que se caracterizaran por su flexibilidad y operaran como laboratorios para experimentar nuevos métodos de impartir los cursos de Estudios Generales. Dentro de su proyecto se habla de fusionar el curso de Historia de la Cultura con Filosofía y dar una sola materia, llamada Humanidades, enfocada en el pensamiento latinoamericanoli.

Más concreto es el proyecto de María Eugenia Dengo y su propuesta sobre las carreras de educación ofrecidas en su plan de Colegios Universitarios Regionales. Utilizando las sedes regionales de la Escuela Normal, Dengo propone:

"Del plan de estudios de las Escuelas Normales se aprovecharía el "Castellano," convirtiéndolo en materia de Estudios Generales, como así natura instrumental indispensable en la adecuada formación de todo profesional. Además, podría ensayarse la tesis de dar a los Estudios Generales una modalidad más dinámica, mediante el ofrecimiento de un solo curso central de "Humanidades", el cual integraría los contenidos histórico - culturales y filosóficos y la aplicación de análisis literario a los mismos a base del estudio de obras fundamentales en la historia literaria y del pensamiento. Colateralmente se organizarían los cursos científicos 
Especial: Profesores de Estudios Generales investigan

básicos: Matemáticas, Biología, Física, Química, Sociología, Psicología, según el área de escogencia del estudiante."lii

Así, en sintonía con Monge Alfaro, dentro de este plan de estudios el Curso Integrado de Humanidades sería fusionado en una sola clase. Igualmente, los cursos científicos del ciclo de Educación Básica (Biología, Química, Matemática) serían aprovechados en cada especialidad.

La formación de docentes se obtendría de dos maneras. Para maestros de primaria se coordinaría junto a Estudios Generales la formación de este tipo de educadores en tres años. Para profesores de secundaria, el objetivo del plan era alcanzar un profesorado en enseñanza media en tres años, con la posibilidad de culminar un grado universitario en la sede central.

Finalmente, en San Ramón se aprueba el funcionamiento de 13 asignaturas a cargo de 8 docentes ${ }^{\text {liii }}$, siendo Fundamentos de Biología el primer curso impartido. En la fuente consultada se puede inferir que los cursos del Departamento de Estudios Generales impartidos serían exclusivamente los necesarios para la formación de los educadores, motivo por el cual no se ofrecía el ciclo de Educación Básica completo. Esto se puede comprobar por la composición del estudiantado, en donde destacan docentes ya activos que buscaban su especialización, y en la presión ejercida por la Juventud en Defensa del Centro Regional de San Ramón. Esta organización buscó ampliar el ciclo de Estudios Básicos y así garantizar que los estudiantes de este centro pudieran optar por su traslado a la Ciudad Universitaria Rodrigo Facio a continuar con la carrera que desearan, ampliando las posibilidades académicas más allá de las carreras de Educación, como estaba contemplado en el plan originaliv ${ }^{\text {liv }}$ El éxito de esta movilización se puede apreciar en el hecho de que los Estudios Generales, como se analizará más adelante, fueron el germen de la creación de los restantes centros regionales, ofreciendo en todos los casos el ciclo completo de Educación Básica.

\section{(c) (i) (2)}

La Revista Estudios es editada por la Universidad de Costa Rica y se distribuye bajo una Licencia Creative Commons Atribución-NoComercial-CompartirIgual 3.0 Costa Rica. Para más información envíe un mensaje a 
Especial: Profesores de Estudios Generales investigan

Es importante resaltar el hecho que estos cursos generales se tomaron como la base de cualquier carrera universitaria de la UCR y aún con carreras cortas o intermedias, estos estuvieron presentes. Esto permite concluir al menos dos cosas, primero, la visión humanista de la Universidad se impregnó desde el inicio en su programa de regionalización y segundo, al consolidarse este programa, esta base común se convirtió en una herramienta que permitió la movilización estudiantil entre recintos. Finalmente, desde un punto de vista administrativo, la regionalización quedó ligada a los Estudios Generales con la administración del primer centro regional por parte de la Facultad de Ciencias y Letras y la Dirección del Departamento de Estudios Generales.

Por los motivos antes expuestos, la entrada en funciones del Centro Regional de Liberia se retrasó hasta 1972. Así, después de San Ramón, el otro recinto en crearse fue el Centro Regional Atlántico en Turrialba y es su proceso de formación el que muestra con mayor evidencia la importancia que tomaron los Estudios Generales en la regionalización universitaria.

La presencia de la Universidad en Turrialba tiene su origen en la organización de la comunidad en torno al Círculo de Aficionados a la Filosofía, agrupación que se reunía en el Centro Cultural John F. Kennedy y que periódicamente convocaba coloquios y conferencias con docentes de Humanidades de la UCR. Este grupo es el núcleo que posteriormente formaría el Comité Pro Centro Regional Universitario e iniciaría una campaña en los medios de comunicación locales para incentivar a la comunidad turrialbeña a apoyar la iniciativalv.

Esta organización logra un importante impulso político con la aprobación de una moción en la Municipalidad de Turrialba para la creación de la Comisión Pro Escuela de Estudios Generales. Este punto es de suma importancia, pues el nombre que adquiere la comisión municipal equipara la creación del centro regional universitario con la creación de un espacio en donde se impartan los 
ISSN 1659-3316

Especial: Profesores de Estudios Generales investigan

Estudios Generales. Si el origen de los centros de San Ramón y Liberia estaba ligado a proyectos muy concretos, la emergencia de titulación de docentes y la creación de una escuela de agricultura respectivamente, Turrialba será el primer ensayo de regionalización en donde los Estudios Generales son su principal motor.

Lo anterior se comprueba en algunas de las acciones de esta Comisión. Por ejemplo, a finales del año de 1970 comunicaron, en los actos de graduación de los liceos locales, la posibilidad de que estos nuevos graduados no tuvieran que viajar hasta la Ciudad Universitaria Rodrigo Facio a recibir los Estudios Generales, pues la posibilidad de que lo hicieran al año siguiente en Turrialba era grande $^{\text {Ivi }}$. Igualmente, esta Comisión redactó el "Documento para la Fundación del Centro Universitario de Turrialba," el cual fue enviado al Consejo Universitario. Para los intereses de esta investigación se destaca lo siguiente:

"Necesitamos que la UNIVERSIDAD DE COSTA RICA (sic) abra una Escuela de Estudios Generales en Turrialba que permita a los estudiantes de este Cantón (sic) y a los de Juan Viñas, Siquirres, Guápiles, Paraíso, Limón y Cartago, tomar en esta Escuela las materias de humanidades, contribuyendo de esta forma a resolver el grave problema de cupo de la Ciudad Universitaria Rodrigo Facio.

La Escuela de Estudios Generales de Turrialba será el primer paso hacia el Centro Universitario de Turrialba, que esperamos esté dentro de dos años."vvii

Al menos dos datos importantes son brindados por el texto anterior. El primero es la estrategia de utilizar los Estudios Generales como el primer paso para la consolidación de un Centro Regional, destacando así la importancia que tuvieron estos en el proceso de regionalización universitaria. El segundo es la estrategia de utilizar estos centros como puntos para descongestionar la matrícula de la Sede Central, al asumir la tarea de brindar el primer paso de una carrera universitaria.

La evolución del proyecto lleva a la Rectoría a conformar una Comisión Especial, que evaluaría las condiciones materiales en Turrialba, específicamente 
Especial: Profesores de Estudios Generales investigan

las aulas del Colegio Nocturno y el auditorio del Centro Cultural John F. Kennedy, para poder impartir lecciones universitarias en esos espacios. En los viajes realizados por esta comisión a Turrialba se destaca la asistencia del director del Departamento de Estudios Generales, Chester Zelaya y los coordinadores de las cátedras de Castellano, Historia de la Cultura y Filosofía. Finalmente el dictamen de este grupo académico es positivo.

Después de superar varias dificultades, como la oposición de varios miembros del Consejo Universitario y el dictamen de esperar hasta las resoluciones del III Congreso Universitario para considerar la apertura o no de un Centro Regional, se dio la inauguración de los Estudios Generales en Turrialba en agosto de 1971, bajó la denominación de "Cursos del Departamento de Estudios Generales en Turrialba". Estos fueron administrados directamente por el Departamento de Estudios Generales de la Facultad de Ciencias y Letras, además los docentes que impartieron los cursos viajaban desde San Pedro, razón por la cual estos se brindaron en horario nocturno. Esto motivó el traslado de las lecciones del Colegio Nocturno al Colegio Clodomiro Picado. Hacia 1973 ya 320 alumnos recibían lecciones en 20 cursos de Biología, Matemáticas y Estudios Generales ${ }^{\text {lviii. }}$.

La experiencia de estos cursos de Humanidades y Ciencias pudo ser compartida por personas que no eran alumnos, pero que podían ser parte del auditorio en las conferencias públicas realizadas por los docentes universitarios. A propósito, Gerardo Contreras Álvarez recuerda:

"Conocía un poco de esta experiencia pedagógica, pues en 1971 se inició la enseñanza universitaria de las Humanidades en Turrialba, aunque no éramos estudiantes universitarios, se nos invitaba a las conferencias magistrales que impartían don Constantino Láscaris, don Carlos Caamaño, señora Hilda Chen Apuy y otros. Fue una gran oportunidad de conocer otros ámbitos del conocimiento."lix

\section{(c) (7) (2)}

La Revista Estudios es editada por la Universidad de Costa Rica y se distribuye bajo una Licencia Creative Commons Atribución-NoComercial-CompartirIgual 3.0 Costa Rica. Para más información envíe un mensaje a 
Especial: Profesores de Estudios Generales investigan

Ya Dengo proponía que los centros regionales debían lograr un vínculo con la comunidad a través de conferencias magistrales y charlas abiertas al públicolx. De esta manera se esperaba que la influencia de la Universidad se manifestara en la región más allá de las aulas.

A pesar de los expresado por el Consejo Universitario "en el entendido que esto no constituye un compromiso para que en el futuro se conviertan dichos estudios en un Centro Regionaplxi, la realidad fue que la apertura de los cursos impulsó la creación del Comité de Desarrollo Universitario y pavimentó el camino hacia la consolidación del Centro Regional del Atlántico, decretado en 1974.

Después de este proceso en Turrialba, los Estudios Generales tomaron un papel protagónico en la apertura de las restantes sedes y recintos universitarios. En Guanacaste, una vez superados los problemas que atrasaron su apertura, en 1972 el inicio de operaciones del Centro Regional de Liberia ofreció el ciclo completo de Educación General Básica con tres materias de Humanidades y cursos de Biología, Matemática y Física Ixii. En el Pacífico Central, en 1975, inició labores la División Universitaria de Puntarenas, dependencia del Centro Universitario de San Ramón, ofreciendo los cursos de Estudios Generales ${ }^{\text {liii }}$. En Limón, a partir de 1975, operó una división del Centro Regional del Atlántico ofreciendo los Estudios Generales y el programa de Transferencia hasta $1980^{\text {Ixiv }}$. Tomando en cuenta que estos centros regionales operaron en sus primeros años en una infraestructura sumamente restringida y con una oferta académica igualmente limitada, parece ser que estos iniciaron labores como espacios para brindar la oportunidad de completar el requisito de los Estudios Básicos a estudiantes de zonas alejadas del Valle Central que después optaron por su traslado a la Sede Central.

Este último punto se pude evidenciar en el Informe de Rectoría presentado en 1979 - 1980, en la sección escrita por Fernando Durán Ayanegui, Vicerrector de Docencia. Ahí se explicitan toda una serie de medidas tomadas por las 
Especial: Profesores de Estudios Generales investigan

autoridades universitarias para fiscalizar el traslado de estudiantes de un recinto a otro dentro de la UCR. Esta normativa se centra en el derecho de un estudiante de cursar la mayor cantidad posible del tramo de su carrera en un recinto regional y tener la opción de trasladarse a la Ciudad Universitaria Rodrigo Facio para completar sus estudios.

Aquí es donde se establece la tipología de carreras con cupo y carreras sin cupo, descrita con anterioridad. Lo importante es que las carreras sin cupo podían ser matriculadas por un estudiante en cualquiera de los recintos de la Universidad, sin necesidad que dicha carrera fuera ofertada en el recinto escogido. De esta manera, los estudiantes matriculados podían avanzar todo lo posible en un recinto antes de optar por trasladarse a la Sede Central a terminar su formación. Esto hace suponer que muchos estudiantes matriculaban en centros regionales esperando avanzar todo lo posible en los planes de estudios de una carrera de su gusto. Tomando en cuenta que la Educación General Básica era ofertada en todos los centros regionales y la oferta académica de estos se reducía a carreras educativas y técnicas, parece ser que la estrategia de matricular en estos recintos para cumplir con el requisito de completar este ciclo de Estudios Básicos y después trasladarse, fue muy utilizada y alimentó, en una medida que aún debe determinarse, la matricula reportada por la regionalización en sus primeros años de existencia.

Lo anterior puede confirmarse con varias afirmaciones emitidas por la Vicerrectoría de Docencia. Así, dentro de la discusión surgida por la abolición de las precarreras ocurrida en 1975 y su posible impacto en las distintas unidades académicas, el vicerrector Guillermo Chaverri Benavides apunta que:

\section{(c) (i) (2)}

La Revista Estudios es editada por la Universidad de Costa Rica y se distribuye bajo una Licencia Creative Commons Atribución-NoComercial-CompartirIgual 3.0 Costa Rica. Para más información envíe un mensaje a 


\section{Especial: Profesores de Estudios Generales investigan}

"La medida que estoy comentando perjudicó enormemente a los Centros Regionales, puesto que se dedicaban en buena parte a ofrecer precarreras. Para evitar el perjuicio el Consejo Universitario estableció la política para traslados y también fijó cuotas en los Centros en las carreras con cupo restringido de la Sede Central. Así un estudiante que ingresa a un Centro Regional forma parte de la cuota de una carrera de la Sede Central y se traslada a ella cuando termina el tramo de asignaturas que el Centro le ofrece.",|xv

Las normas que cita el vicerrector se concretizan en el informe presentado en 1979-1980, en donde otro vicerrector, Durán Ayanegui, da noticia sobre su operación:

"La aplicación de estas normas y definiciones, así como una serie de medidas administrativas conexas, nos ha permitido reducir a un mínimo el problema presentado hasta ahora por los estudiantes que después de uno o más años de vida académica no han escogido aún su carrera, situación que en los Centros Regionales había alcanzado proporciones alarmantes"|xvi.

Estas citas sugieren que la advertencia hecha en el Consejo Universitario una década antes se cumplió y los centros regionales se convirtieron en espacios en donde estudiantes buscaban concluir la Educación General Básica, sin perspectivas de concluir una carrera en dicho centro y utilizándolos como una plataforma para cumplir requisitos del plan de estudios con el objetivo de trasladarse más adelante a la sede central de la Universidad. Este proceso se aceleró con la decisión del Consejo Universitario de abolir la precarrera y permitir a los alumnos matricular cualquier carrera de su elección en cualquiera de los recintos universitarios. Sumado a lo anterior parece ser que la lenta consolidación de carreras propias, la falta de desconcentración de carreras más allá de las educativas y la falta de oferta de una titulación mayor a un técnico, incidieron en que la posibilidad de completar toda una carrera en un centro regional no fuera tan atractiva. Contrastado con el éxito de matrícula que significó la regionalización en su primera década, alrededor del 16,7 por ciento del total de la UCR hacia 1979lxvii, hace suponer que la mayoría eran estudiantes temporales, esperando "sacar las Generales".

\section{(c) (i) (-)}

La Revista Estudios es editada por la Universidad de Costa Rica y se distribuye bajo una Licencia Creative Commons Atribución-NoComercial-CompartirIgual 3.0 Costa Rica. Para más información envíe un mensaje a revistaestudios.eeg@ucr.ac.cr. 


\section{Conclusiones}

\section{Especial: Profesores de Estudios Generales investigan}

La educación superior en Costa Rica experimentó un periodo de profundos cambios en las décadas de 1960 y 1970. A nivel interno, estas transformaciones están relacionadas con la modernización sufrida por la sociedad costarricense, la expansión de los servicios prestados por el Estado y el cambio en el modelo productivo nacional, todo lo anterior contextualizado desde una perspectiva desarrollista. De esta manera, la educación universitaria se adaptó a las nuevas demandas de la sociedad costarricense y se colocó en sintonía con el proyecto desarrollista. En el caso de la UCR, esta modernizó y adaptó su estructura a las nuevas exigencias con las decisiones tomadas en el III Congreso Universitario entre 1971 - 1973 e impulsó un programa de regionalización que intentó conectar desarrollo regional con regionalización universitaria.

A nivel externo, estas décadas se contextualizan dentro de la Guerra Fría y la estrategia de Estados Unidos para contener al comunismo en América Latina. Así, esta nación desarrolló un vigoroso proyecto de cooperación para lograr este objetivo, en donde se destaca su política educativa. Las universidades estatales centroamericanas fueron parte de este proceso, manifestado en su integración dentro del CSUCA y la implementación de planes para departamentalizar sus estructuras. De igual manera, la estandarización de estos centros de enseñanza pasaba por la aplicación conjunta de un ciclo de Educación General, inspirado en el modelo estadounidense, común a todos los estudiantes matriculados. En distintos niveles, este modelo fue aplicado como política de la CSUCA. La consecuencia común más palpable es la radicalización política de las generaciones de estudiantes que recibieron este tipo de educación.

Aplicados en distintas modalidades, desde la Reforma de 1957 la propuesta de Estudios Generales fue exitosa en la UCR. Con el inicio del programa de regionalización en 1968 y la expansión de la Universidad fuera del

\section{(c) (i) (2)}

La Revista Estudios es editada por la Universidad de Costa Rica y se distribuye bajo una Licencia Creative Commons Atribución-NoComercial-CompartirIgual 3.0 Costa Rica. Para más información envíe un mensaje a 


\section{Especial: Profesores de Estudios Generales investigan}

Valle Central, el modelo de Estudios Generales también se regionalizó. Aunque en un principio estos se restringieron a una modalidad adaptada a las carreras cortas, por presión estudiantil el ciclo de Estudios Básicos se ofreció de manera completa, dando así la oportunidad a los alumnos de los centros regionales de cumplir con este requisito y poder trasladarse a la sede central a completar una carrera. Esto tuvo como consecuencia que muchos estudiantes que matricularon en estos centros no tuvieran contemplado finalizar una carrera ahí y los utilizaran como trampolín para trasladarse a la Ciudad Universitaria Rodrigo Facio.

\section{Bibliografía}

Andreu, Cristopher (2015). Historia institucional de la Facultad de Ciencias y Humanidades de la Universidad de El Salvador 1948-1972. (Tesis de Licenciatura, Universidad de El Salvador) Recuperado de http://ri.ues.edu.sv/15161/1/14102790.pdf

Araya Pochet, Carlos (1990). La Universidad de Costa Rica: 1972-1990. Transformaciones, crisis y perspectivas. Revista de Historia, (21-22), 231-261.

(1994). La Universidad Nacional y la educación superior estatal en veinte años de historia, 1973-1993. Heredia: FUNDAUNA.

Barahona Jiménez, Luis. (2015). La Universidad de Costa Rica, 1940-1973. San José: Editorial Universidad de Costa Rica.

\section{(C) $(00$}

La Revista Estudios es editada por la Universidad de Costa Rica y se distribuye bajo una Licencia Creative Commons Atribución-NoComercial-CompartirIgual 3.0 Costa Rica. Para más información envíe un mensaje a 
Especial: Profesores de Estudios Generales investigan

Calderón Padilla, Rutilia (2011). El crecimiento y desarrollo de la educación superior en Honduras, una perspectiva desde la UNAH. Innovación Educativa, 11, (57), 81-89.

Castro Sánchez, Silvia (2012). Costa Rica frente a la regionalización de la educación superior. El primer centro universitario regional en San Ramón, Alajuela. San Ramón: Coordinación de Investigación, Sede Occidente, Universidad de Costa Rica.

Centro de Capacitación y Promoción para la Democracia (CECADE) (2013). Tiempos de audacia. La mass media de una guerrilla. San Salvador: CECADE.

Chavarría Alfaro, Gabriela (2012). Los Estudios Generales en las universidades públicas de Centroamérica: 1950 - 1970. San José: ALICAC.

Chinchilla Gutiérrez, Sara (2011). Estudios Generales en sus hechos. San José: EDITORAMA.

Contreras Álvarez, Gerardo (2015). Vivir los Estudios Generales. Estudios, (30), 14.

Cruz De Lemos, Vladimir de la (2015) ¿Qué significó el paso por Estudios Generales? Estudios, (30), 1-7.

Fonseca Tortós, Emilia (2007). Del concepto a la imagen. Cincuentenario de los Estudios Generales en la Universidad de Costa Rica. Reflexiones, 86, (2), 51-64.

\section{(C) $(00$}

La Revista Estudios es editada por la Universidad de Costa Rica y se distribuye bajo una Licencia Creative Commons Atribución-NoComercial-CompartirIgual 3.0 Costa Rica. Para más información envíe un mensaje a 
Especial: Profesores de Estudios Generales investigan

Gamboa Umaña, Luis Enrique (2015). La política pública en la creación de universidades en Costa Rica en el decenio de 1970. (Tesis de doctorado sin publicar). Universidad de Costa Rica, San José, Costa Rica.

Gutiérrez Carranza, Claudio (1977). Informe del Rector 1976-1977. San José: Publicaciones de la Universidad de Costa Rica. Recuperado de http://www.rectoria.ucr.ac.cr/site/wp-content/uploads/2013/11/1976-1977.pdf

(1980). Informe del Rector 1979-1980. San José: Publicaciones de la Universidad de Costa Rica. Recuperado de http://www.rectoria.ucr.ac.cr/site/wp-content/uploads/2013/11/1979-1980.pdf

Hernández Faerrón, Mireya (2013). Semblanza histórica de la Sede Universitaria de Guanacaste, Universidad de Costa Rica. InterSedes, XIV, (29), 152-163.

Hernández Rodríguez, Ana Cecilia (2001). La docencia de las Sedes Regionales de la Universidad de Costa Rica. Informe de la Sede del Pacífico. Manuscrito inédito. Departamento de Docencia Universitaria, Universidad de Costa Rica, San José, Costa Rica.

Lepe Jorquera, Ivone. (2018). La Sede del Caribe: experiencia de su desarrollo en el período 2002-2010. Ponencia presentada el día 18 de abril de 2018 en el I Congreso de Regionalización UCR en Sede Occidente, San Ramón.

Molina Jiménez, Iván (2016). La educación en Costa Rica. Desde la época colonial hasta el presente. San José: EDUPUC.

\section{(C) $(\triangle \Theta$}

La Revista Estudios es editada por la Universidad de Costa Rica y se distribuye bajo una Licencia Creative Commons Atribución-NoComercial-CompartirIgual 3.0 Costa Rica. Para más información envíe un mensaje a 
Especial: Profesores de Estudios Generales investigan (2019). La huelga estudiantil de 1982 y la democratización del Instituto Tecnológico de Costa Rica. Diálogos, 23, (1), 130-150.

Molina Vargas, Silvia Elena (2008). La joven Benemérita Universidad Estatal a Distancia: Institución Benemérita de la Educación y la Cultura. San José: EUNED.

Monge Alfaro, Carlos (1967). Informe del Rector. 1966-1967. San José: Publicaciones de la Universidad de Costa Rica. Recuperado de http://www.rectoria.ucr.ac.cr/site/wp-content/uploads/2014/09/1966-1967.pdf

(1968). Informe del Rector. 1967-1968. San José: Publicaciones de la Universidad de Costa Rica. Recuperado de http://www.rectoria.ucr.ac.cr/site/wp-content/uploads/2014/09/1967-1968.pdf

(1969). Informe del Rector. 1968-1969. San José: Publicaciones de la Universidad de Costa Rica. Recuperado de http://www.rectoria.ucr.ac.cr/site/wp-content/uploads/2014/09/1968-1969.pdf

Pérez, Olga (2014). Entre movimientos y reformas universitarias en Guatemala: de la segunda mitad del siglo XX a la actualidad. Estudios Digital, (14), 1-27.

Ramírez Fuentes, José Alfredo (2013). Humanidades, Facultad y Reforma: los años 60 en la Universidad de El Salvador. Revista Humanidades V, (1), 87-109.

Reina Idiáquez, Jorge Arturo (1999). Historia de la UNAH en su época autónoma. Tegucigalpa: Editorial Universitaria.

\section{(C) $(00$}

La Revista Estudios es editada por la Universidad de Costa Rica y se distribuye bajo una Licencia Creative Commons Atribución-NoComercial-CompartirIgual 3.0 Costa Rica. Para más información envíe un mensaje a 
Especial: Profesores de Estudios Generales investigan

Salazar Mora, Orlando (1998). Sede Regional del Atlántico. San José: Editorial Universidad de Costa Rica.

Stansifer, Charles \& Bozzoli, María Eugenia (2010). La Universidad de Costa Rica y la Universidad de Kansas: su extraordinaria relación de cooperación académica. San José: Editorial de la Universidad de Costa Rica.

Tarracena Arriola, Arturo. Comunicación personal, 20 de agosto 2019.

Trujillo Molina, Ricardo. Comunicación personal, 13 de setiembre 2019.

Tunnermann Berheim, Carlos (2008). La educación superior en Nicaragua. Avaliação: Revista da Avaliação da Educação Superior, 13 (2) 337-367.

Villanueva Monge, Zarela (2015). Estudios Generales: una ventana al mundo. Estudios, (30), 1-3.

'Molina, 2016. Pág. 412.

ii Ibidem. Pág. 323.

iii Monge, 1968. Pág. 22.

iv De la Cruz, 2015. Pág. 4.

Ibidem.

vi Villanueva, 2015. Pág. 2.

vii De la Cruz, 2015. Pág. 4.

viii Villanueva, 2015. Pág. 3.

${ }^{\text {ix }}$ Araya, 1990. Pág. 232 y ss.

x Tarracena, 2019.

xi Ramírez, 2013. Pág. 102.

xii Trujillo, 2019 y Tarracena, 2019.

xiii CECADE, 2013.

${ }^{\text {xiv }}$ Araya, 1994. Pág. 36.

xv Gamboa, 2015. Pág. 111.

xvi Molina, 2008. Págs. 25 y ss.

xvii Molina, 2016. Pág. 398.

xviii Molina, 2019.

La Revista Estudios es editada por la Universidad de Costa Rica y se distribuye bajo una Licencia Creative Commons Atribución-NoComercial-CompartirIgual 3.0 Costa Rica. Para más información envíe un mensaje a revistaestudios.eeg@ucr.ac.cr. 
${ }^{\text {xix }}$ Araya, 1990. Págs. 235 y ss.

${ }^{x x}$ Molina, 2016. Pág. 410.

xxi Castro, 2012.

xxii Monge, 1968. Pág. 21.

xxiii Ibidem. Pág. 22.

xxiv Monge, 1969. Pág. 71.

${ }^{x \times v}$ Araya, 1994.

xxvi Monge, 1967. Pág. 113.

xxvii Monge, 1969. Pág. 70 y ss.

xxviii Monge, 1967. Pág. 114.

xxix Chavarría, 2012. Págs. 48 y ss.

${ }^{x \times x}$ Chinchilla, 2011.

xxxi Ibidem.

xxxii Barahona, 2015. Capítulo IV.

xxxiii Stansifer y Bozzoli, 2010. Págs. 47 y ss.

xxxiv Pérez, 2014. Pág. 8.

xxxv Calderón, 2011. Pág. 82.

xxxvi Tunnermann, 2008. Pág. 343.

xxxvii Chavarría, 2012. Pág. 39.

xxvviii Andreu, 2015. Págs. 51 y ss.

${ }^{x x x i x}$ Reina, 1999. Tomo uno. Pág. 276 y ss.

${ }^{x l}$ Chavarría, 2012. Pág 30-31 y Stansifer y Bozzoli, 2010. Pág. 67 y ss.

${ }^{x l i}$ Stansifer y Bozzoli, 2010. Pág. 67 y ss.

xlii Andreu, 2015. Pág. 53.

xliii Citado en Chavarría, 2012. Pág. 80.

xliv Chinchilla, 2011.

${ }^{x / v}$ Fonseca, 2007. Pág. 54.

xlvi Molina, 2016. Pág. 395.

xlvii Gutiérrez. 1977, Pág. 30.

xlviii Ibidem. Pág. 28 y ss.

${ }^{x i l x}$ Salazar, 1998. Pág. 31 y ss.

'Ibidem. Pág. 39.

li Ibidem.

lii Monge, 1967. Pág. 113 y ss.

liii Castro, 2012. Pág. 49.

liv Ibidem.

Iv Salazar, 1998. Pág. 55 y ss.

Ivi Ibidem.

Ivii Ibidem.

Iviii Ibidem. Pág. 88.

lix Contreras, 2015. Pág. 2.

${ }^{\mathrm{x}}$ Monge, 1967. Pág. 115.

Ixi Salazar, 1998.

Ixii Hernández, 2013.

Ixiii Hernández, 2001. Pág. 8.

Ixiv Lepe, 2018. Pág. 7.

Ixv Gutiérrez, 1980. Pág. 30.

La Revista Estudios es editada por la Universidad de Costa Rica y se distribuye bajo una Licencia Creative Commons Atribución-NoComercial-Compartirlgual 3.0 Costa Rica. Para más información envíe un mensaje a revistaestudios.eeg@ucr.ac.cr. 
Revista Estudios

Febrero 2020

ISSN 1659-3316

Fonseca Zúñiga Edgardo

Especial: Profesores de Estudios Generales investigan

Ixvi Ibidem.

Ixvii Molina, 2016. Cuadro 8.10, Pág. 403

\section{(c) (i) (2) (2)}

La Revista Estudios es editada por la Universidad de Costa Rica y se distribuye bajo una Licencia Creative Commons Atribución-NoComercial-CompartirIgual 3.0 Costa Rica. Para más información envíe un mensaje a revistaestudios.eeg@ucr.ac.cr. 